\title{
The Pattern of Presentation and Incidence of Tuberculosis in Patients on Chronic Hemodialysis
}

Pavan M, MD*

\author{
Associate Professor, Division of Nephrology, Department of Medicine, Adichunchanagiri Institute of Medical Sciences, B. G. Nagara, Karnataka State, \\ India
}

DOI: $10.36347 /$ sjams.2020.v08i09.029

| Received: 11.09.2020 | Accepted: 18.09.2020 | Published: 20.09.2020

*Corresponding author: Pavan M

Abstract

Original Research Article

Objective: The incidence of tuberculosis (TB) among dialysis population is much higher than general population. Immunosuppression induced by end-stage renal disease (ESRD) modified the clinical presentation of TB, resulting in atypical signs and symptoms, and a more frequent extrapulmonary presentation. This study was undertaken to determine the pattern of presentation and incidence of TB in ESRD patients. Methods: This was a prospective observational study done using 200 ESRD patients who are on chronic maintenance hemodialysis (HD) at Adichunchanagiri Institute of Medical Sciences, BG nagara, India. TB was diagnosed using clinical, radiological, biochemical, microbiological, and histological findings. Results: The incidence of TB was found to be $12 \%$. It was found to be commoner in females, commonest in the age group 40-34 years. Pleural effusion was the commonest type of TB found $(50 \%)$. The incidence of extrapulmonary TB was $87.5 \%$. There was a high incidence of TB during the early years of initiation of HD. Patients with TB had a statistically significant low BMI compared to non-TB patients $\left(18.42 \mathrm{~kg} / \mathrm{m}^{2}\right.$ Vs. $\left.22.63 \mathrm{~kg} / \mathrm{m}^{2} . P<0.001\right)$. TB had a significant impact on mortality among HD patients. Conclusion: Patients with ESRD, on chronic maintenance HD are at increased risk for pulmonary and extra pulmonary tuberculosis and should be diagnosed with high index of suspicion. The TB infected patients generally presented worse mortality rates than the non-TB infected patients.

Keywords: End-stage renal disease, hemodialysis, mortality, tuberculosis.

Copyright @ 2020: This is an open-access article distributed under the terms of the Creative Commons Attribution license which permits unrestricted use, distribution, and reproduction in any medium for non-commercial use (NonCommercial, or CC-BY-NC) provided the original author and source are credited.

\section{INTRODUCTION}

Tuberculosis (TB) is a widespread infectious disease, most of which occurring in developing countries. People in the developing world contract tuberculosis because of a poor immune system [1]. Endstage renal disease patients who are on chronic maintenance hemodialysis will have poor cell mediated immunity. This has led to increased TB rates in dialysis population [2]. An individual who is on hemodialysis has 6.9 to 52.5 times increased risk of developing TB compared to healthy individual [3].

The presentation of TB in dialysis patients varies from the normal, both in clinical features and investigation findings. Also there is a rise in the extrapulmonary presentation of TB [3]. Data on the incidence and prevalence of TB in dialysis patients in India is scanty. We undertook this study to determine the pattern of presentation of TB in dialysis population and to ascertain the incidence of TB in dialysis patients.

\section{Patients And Methods}

Study Setting: The study was done simultaneously at Adichunchanagiri Institute of Medical Sciences, B.G. nagar between January 2016 and December 2018. The patients for the study were recruited from the dialysis unit of both the hospitals.

Sample Size: 100 patients who are on chronic maintenance hemodialysis were studied. Sampling Method: All consecutive ESRD patients who are on maintenance HD aged 18 years and above, who presented to the dialysis department within the study period and who met the selection criteria, were recruited into this study.

\section{Selection CRiteria Inclusion Criteria}

(1) All patients who are on maintenance HD aged 18 years and above who presented to the dialysis department within the study period. 
(2) All patients who consented to participate in the study.

\section{Exclusion Criteria}

(1) All chronic kidney disease patients who are not on dialysis.

(2) Patients who did not consent to join the study.

(3) All ESRD patients below age 18 years.

\section{Method of Data Collection}

All subjects that met the inclusion criteria were clinically assessed. That involved detailed history taking and a physical examination. A designed questionnaire was used as the study instrument. All subjects gave informed consent for participation in the study, which was approved by the human ethics committee. All of the procedures were in accordance with the Helsinki Declaration of 1975.

The above information with the subject's biodata and the results of the following investigations were entered into the questionnaire.

(a) Full blood count and Erythrocyte Sedimentation Rate (FBC + ESR).

(b) Chest radiograph.

(c) Sputum acid and alcohol fast bacilli (AAFB).

(d) Smear and culture specimens from sputum, peritoneal, pleural, pericardial or cerebrospinal (CSF) fluid.

(e) Related tissue biopsy sampling (lymph node, peritoneal, pleural or any other tissue involved)

\section{Method of Data Analysis}

The Microsoft excel computer software was used to record the data. The statistical package for social sciences (SPSS) version 13.0 was used to analyse them. Frequency tables were drawn to show the distribution of data within variables. Contingency tables were drawn to compare two discrete variables.

\section{RESULTS}

A total of 200 patients were recruited in the study. The ages of the respondents ranged between 19 and 74 years. The mean age was 57.2 years. Of all the 200 patients studied, $123(37.3 \%)$ were males. The remaining 77 patients $(62.7 \%)$ were females. Twentyfour $(12 \%)$ of the 200 -dialysis patients were diagnosed with TB. Among the patients diagnosed with TB, 14 of them were females while the remaining 10 are males, giving a male to female sex ratio of 1: 1.4 (Table 1). The incidence of TB is highest among the age group 40-49 years ( 9 cases), followed by the age group 50-59 years (6 cases). The lowest incidence is seen in age groups less than 20 years ( 1 case) and more than 60 years (2 cases) (Figure 1).

The commonest presentation was tubercular pleural effusion, with 12 patients $(50 \%)$ out of the 24 patients with TB infections. The least common was disseminated TB (1 patients $(4.1 \%))$. Six patients $(25 \%)$ were found to have tubercular adenitis, while 3 patients $(12.5 \%)$ had pulmonary tuberculosis and 2 patients (8.3\%) had abdominal TB (Figure 2).

The most common symptom presented by the TB infected respondents was weight loss with 23 respondents $(95.8 \%)$. This was followed by fever, which occurred in 20 respondents $(83.3 \%)$. Difficulty in breathing was found in 18 respondents $(75 \%)$. Cough was found in 15 respondents $(62.5 \%)$. A total of 11 patients had night sweat $(45.8 \%)$. Sputum production occurred in 4 respondents $(16.6 \%)$, while hemoptyasis was found in 2 respondents $(8.3 \%$ ) (Table 2$)$

There was a significant relationship between low body mass index (BMI) and $\mathrm{TB}$ in dialysis population (Figure 3). The mean BMI of patients without TB was $22.63 \mathrm{~kg} / \mathrm{m} 2$, while the mean BMI of patients with TB was $18.42 \mathrm{~kg} / \mathrm{m} 2, P$ value $<0.001$. Duration on dialysis had a bearing on the incidence of TB. When patients were on dialysis for $<1$ year, we had 12 patients out of $24(50 \%)$ with diagnosis of TB. TB was diagnosed in 8 patients between 1-3 years of HD and in 4 patients with $>4$ years of HD (Table 3 ). No statistically significant association was found between the TB and non-TB groups with respect to other predisposing factors for TB such as diabetes, retroviral disease, smoking and occupational exposure.

Furthermore, TB contributed significantly to the mortality among hemodialysis patients. On applying Kaplan Meyer survival analysis between status of TB and days of mortality, a significant survival benefit was observed $(p<0.001)$ in non-TB patients (Figure 4$)$.

\section{DiSCUSSION}

ESRD patients who are on chronic maintenance HD are prone to many infections including TB because of poor cell mediated immune response [4]. In our study the incidence of TB among HD patients was $12 \%$. This agrees with the study by Ghulam Hassan Malik et al. who demonstrated that $14.5 \%$ of the cases were found to have TB [5]. Diagnosing TB in dialysis population is challenging because of its varied presentation, non-specific symptoms and high incidence of extrapulmonary involvement [6]. Extrapulmonary involvement has been reported between $38-80 \%$ among dialysis population, whereas in general population extrapulmonary TB is reported to account for only $4.5 \%$ of the total cases of TB [7]. In our study, the incidence of extrapulmonary TB was $87.5 \%$. We found tuberculous pleural effusion as the most common type of extrapulmonary TB. Classical TB symptoms (cough \& hemoptyasis) in general population are less frequently reported in dialysis patients [8]. These symptoms are reported in an average $22 \%$ of dialysis TB patients [9]. In our study, both of these symptoms were reported in only $8.3 \%$ of patients. The most 
common presenting symptom of TB in our study was weight loss, accounting for $95.8 \%$ of all TB patients. This may probably due to $\mathrm{TB}$, which is a chronic debilitating disease, is coexisting with another chronic inflammatory condition (chronic kidney disease).

The mean body mass index (BMI) of TB infected patients $(20.42 \mathrm{~kg} / \mathrm{m} 2)$, was found to be less than that of the non TB infected patients $(22.63 \mathrm{~kg} / \mathrm{m} 2)$. This is expected as the coexistence of the TB with end stage renal disease will ultimately affect the BMI negatively. In our study, most of the TB cases were diagnosed $(50 \%)$ within first year of dialysis. These results are similar to what was found in the studies done by Erkoc et al. [10]. This may be due to the generalized debility and profoundly depressed cell mediated immunity during the early months of initiation of dialysis. Several studies reported a high mortality of $17 \%$ to $75 \%$ in CKD patients with TB $[11,12]$. In our study, significantly high mortality rate $(70 \%)$ was observed in TB infected patients. Poor nutritional status, delay in diagnosis because of varied presentation, drug toxicity and poor compliance might have led to this increased mortality in these patients.

Limitations of our study includes: a) small number of the study population, b) delay in diagnosing TB due to its varied presentation in HD population and c) ours is only an observational study.

\section{CONCLUSION}

In conclusion, TB is common in hemodialysis patients, and this worsens their clinical status. The presentation of TB in dialysis patients is mostly atypical making diagnosis difficult. The TB infected patients generally presented worse mortality rates than the nonTB infected patients.

Table-1: Clinical characteristics and laboratory findings of the study population

\begin{tabular}{|l|l|}
\hline Parameters & Mean \pm SD \\
\hline Age & $57.2 \pm 6.4$ \\
\hline Sex $($ Male/Female) & $123 / 77$ \\
\hline Laboratory Findings & \\
\hline Hemoglobin $(\mathrm{g} / \mathrm{dL})$ & $10.6 \pm 0.42$ \\
\hline Creatinine $(\mathrm{mg} / \mathrm{dL})$ & $8.62 \pm 4.26$ \\
\hline WBC $\left(/ \mathrm{mm}^{3}\right)$ & $9400 \pm 1600$ \\
\hline Total Protein $(\mathrm{g} / \mathrm{dl})$ & $6.5 \pm 3.7$ \\
\hline Serum Albumin $(\mathrm{g} / \mathrm{dl})$ & $3.02 \pm 0.6$ \\
\hline ESR $(\mathrm{mm} / \mathrm{hr})$ & $74.2 \pm 24.6$ \\
\hline
\end{tabular}

Table-2: Distribution of presenting symptoms of TB among all the study population

\begin{tabular}{|l|l|l|}
\hline Symptom & Frequency & Percentage \\
\hline Weight Loss & 23 & $95.8(\%)$ \\
\hline Fever & 20 & $83.3(\%)$ \\
\hline Difficulty in Breathing & 18 & $75(\%)$ \\
\hline Cough & 15 & $62.5(\%)$ \\
\hline Night Sweat & 11 & $45.8(\%)$ \\
\hline Sputum & 4 & $16.6(\%)$ \\
\hline Hemoptyasis & 2 & $8.3(\%)$ \\
\hline
\end{tabular}

Table-3: Relationship between duration on dialysis and time of diagnosis of TB among study population

\begin{tabular}{|l|l|}
\hline Duration on Dialysis (years) & Diagnosis of TB (Percentage) \\
\hline$<1$ & $12(50 \%)$ \\
\hline $1-3$ & $8(33.33 \%)$ \\
\hline$>3$ & $4(16.66 \%)$ \\
\hline
\end{tabular}




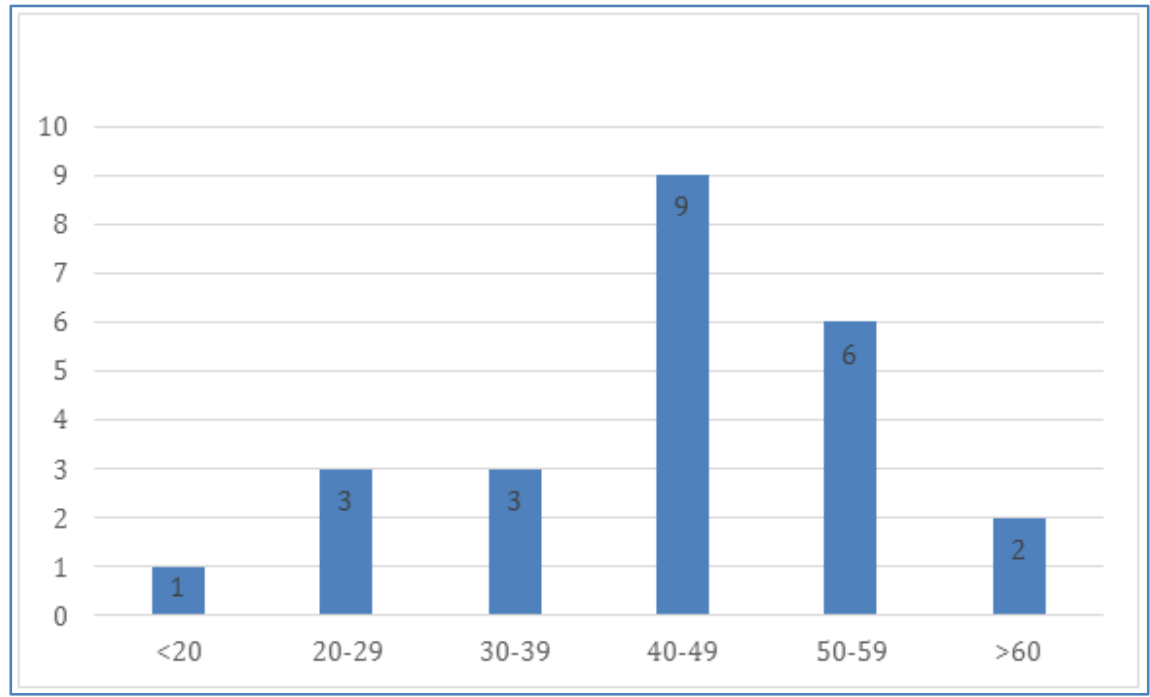

Fig-1: Incidence of TB among different age groups

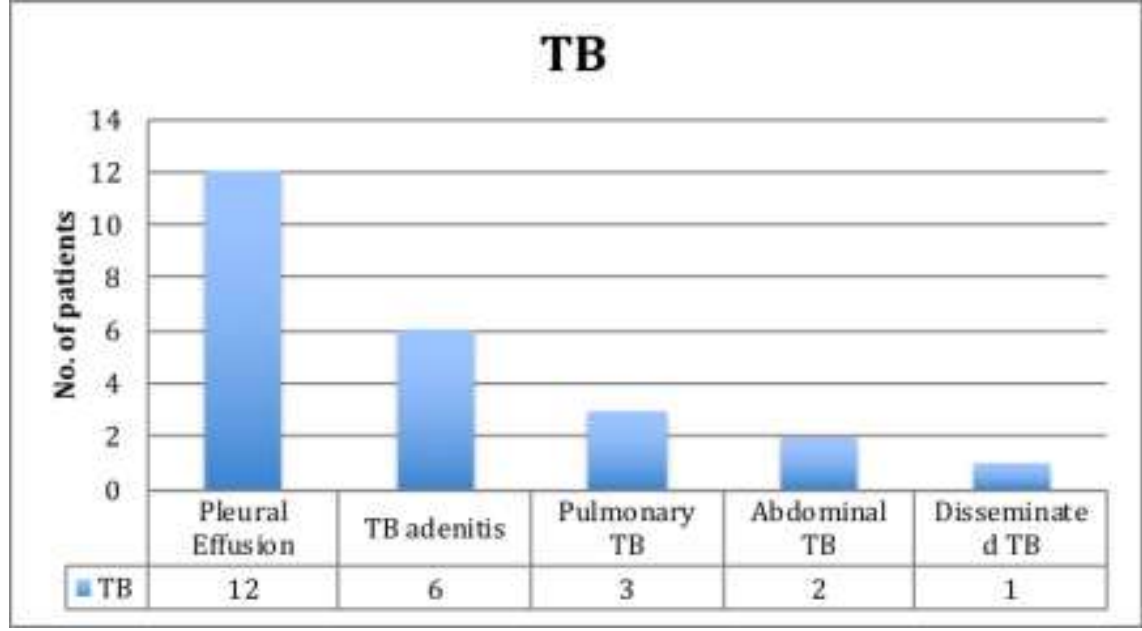

Fig-2: Distribution of the types of TB found in the study population

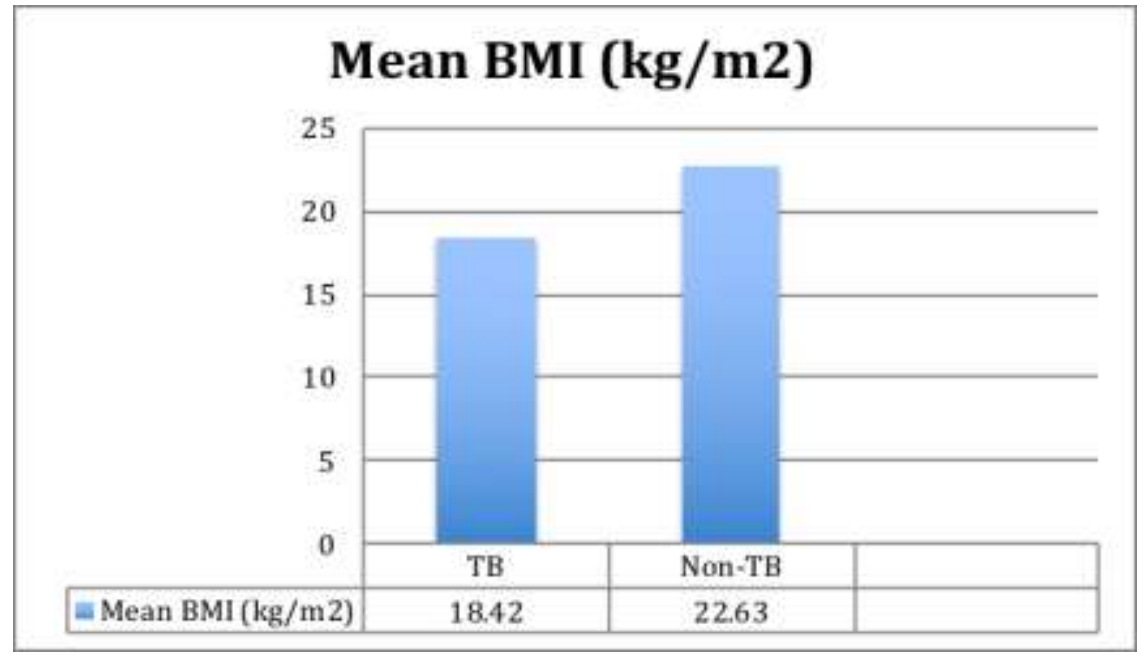

Fig-3: Comparison of BMI between TB and non-TB patients 


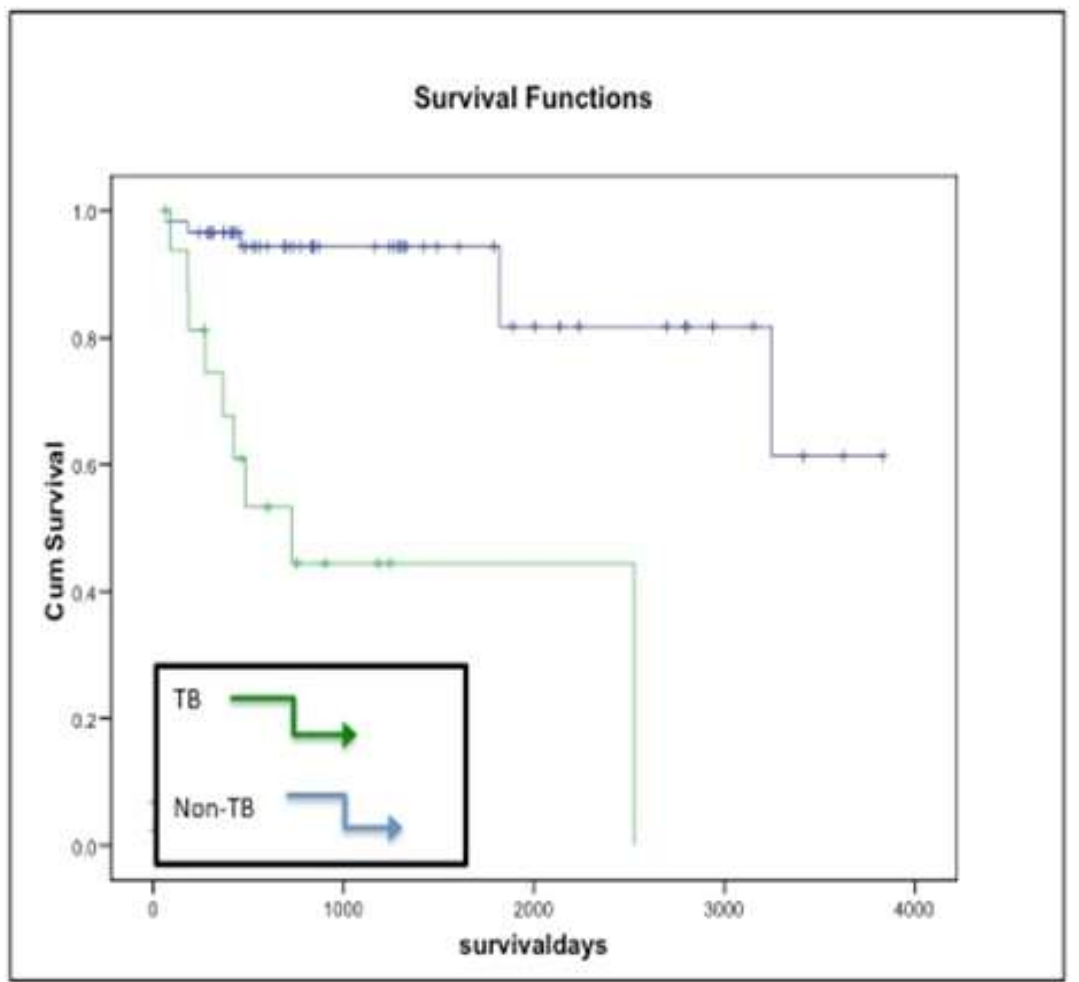

Fig-4: Relationship between TB and Mortality among dialysis patients

\section{IMPLICATION FOR HEALTH POLICY Makers/Practice/Research/Medical Education}

Nephrologists should be aware of the pattern of presentation and the atypical findings of tuberculosis for early diagnosis and treatment among hemodialysis population.

\section{REFERENCES}

1. Lawn SD, Zumla AI. Tuberculosis. Lancet. 2011; 378(9785):57-72.

2. Hussein MM, Mooij JM, Roujouleh $H$. Tuberculosis and chronic renal disease Semin Dial. 2003; 1: 38-44.

3. Siriram SN. Arvind M. Optimal tuberculosis screening of hemodialysis patients. Nephron. 1992; 82: 356

4. Yousef AI, Ismael MF, Elshora AE, Abdou HE. Pulmonary tuberculosis in patients with chronic renal failure at Zagazig University Hospitals. Egyptian Journal of Chest Diseases and Tuberculosis. 2014;63(1):187-192.

5. Malik GH, Al-Mohaya SA, Al-Harbi AS, Kechrid M, Azhari O, Shetia S, Tashkandy MA, Ahmad K, Bhat AW, Want MA, Subramanian PT. Spectrum of tuberculosis in dialysis patients in Saudi Arabia. Saudi Journal of Kidney Diseases and
Transplantation. 2003 Apr 1;14(2):145.

6. Milburn HJ. How should we treat tuberculosis in adult patients with chronic kidney disease? Pol Arch Med Wewn. 2010; 120: 417-422.

7. Pien FD, Younoszai BG, Pien BC. Mycobacterial infections in patients with chronic renal disease. Infect Dis Clin N Am. 2001;15: 851-876.

8. Unsal A, Ahbap E, Basturk T, Koc Y, Sakaci T, Arar AS, Kayabasi H, Sevinc M. Tuberculosis in dialysis patients: a nine-year retrospective analysis. The Journal of Infection in Developing Countries. 2013 Mar 14;7(03):208-13.

9. Chou KJ, Fang HC, Bai KJ, Hwang SJ, Yang WC, Chung HM. Tuberculosis in maintenance dialysis patients. Nephron. 2001;88: 138-143.

10. Erkoc R, Dogan E, Sayarlioglu H, Etlik O, Topal C, Calka F, Uzun K. Tuberculosis in dialysis patients, single center experience from an endemic area. Int J Clin Pract. 2004; 58: 1115- 1117.

11. Klote MM, Agodoa LY, Abbott KC: Risk factors for Mycobacterium tuberculosis in US chronic dialysis patients. Nephrol Dial Transplant. 2006; 21: 3287-3292

12. Segall L, Covic A. Diagnosis of Tuberculosis in Dialysis Patients: Current strategy. Clin J Am Soc Nephrol. 2010; 5(6):1114-22. 